\title{
Preliminary evaluation of SensHand V1 in assessing motor skills performance in Parkinson Disease
}

\author{
Filippo Cavallo, Dario Esposito, Erika \\ Rovini, Michela Aquilano, Maria \\ Chiara Carrozza, Paolo Dario \\ Scuola Superiore Sant'Anna \\ The BioRobotics Institute \\ Pontedera (PI), Italy \\ f.cavallo@sssup.it
}

\author{
Carlo Maremmani \\ Area Health Authority District 1 \\ Neurology Operative Unit \\ Carrara (MS), Italy
}

\author{
Paolo Bongioanni \\ Azienda Ospedaliero-Universitaria Pisana \\ Neuroscience Department, \\ Neurocare Onlus \\ Pisa, Italy
}

\begin{abstract}
Nowadays, the increasing old population $65+$ as well as the pace imposed by work activities lead to a high number of people that have particular injuries for limbs. In addition to persistent or temporary disabilities related to accidental injuries we must take into account that part of the population suffers from motor deficits of the hands due to stroke or diseases of various clinical nature. The most recurrent technological solutions to measure the rehabilitation or skill motor performance of the hand are glove-based devices, able to faithfully capture the movements of the hand and fingers. This paper presents a system for hand motion analysis based on 9-axis complete inertial modules and dedicated microcontroller which are fixed on fingers and forearm. The technological solution presented is able to track the patients' hand motions in real-time and then to send data through wireless communication reducing the clutter and the disadvantages of a glove equipped with sensors through a different technological structure. The device proposed has been tested in the study of Parkinson's disease.
\end{abstract}

Keywords- Hand rehabilitation, hand motion recognition, smart gloves, Parkinson's disease.

\section{INTRODUCTION}

Nowadays, hand injuries affect a high number of people due to the increasing old population $65+$ as well as the pace imposed by work activities. According to some studies, accidents involving hands often are due to mishaps at home or at work, made more serious by the lack or incorrect use of personal protective equipment. In addition to persistent or temporary disabilities related to accidental injuries we must take into account that part of the population suffers from motor deficits of the hands due to stroke or diseases of various clinical nature. As a consequence of the increasing injuries, of the rapid aging of population as well as of disease progression which compromise the human motion capabilities, different researches on glove rehabilitation procedures have been carried out through the development of new technologies. The aim of these studies was to accelerate the healing process wherever possible and to delay the progression of diseases reducing, at the same time, the medical and social cost of a classical rehabilitation path.
An accurate hand motion recognition has been the focus of many research groups to track the main movements of the hand and its approximately 20 major degrees-of-freedom (DoF), studying a wide variety of grasps and postures. The hand motion capturing methods which have been developed find different fields of application such as the control of robotic prosthetic hands [1], methods of gesture recognition [2], applications in biomedical systems [3] and, in particular, hand rehabilitation procedures [4]. The most recurrent technological solution for the rehabilitation of the hand is the implementation of intelligent gloves able to faithfully capture the movements of hand and fingers thus generating a series of data manageable through the development of virtual realities [5]. The combination between smart gloves and virtual realities has led to the study of new services such as systems for distance monitoring and self-rehabilitation [6] or rehabilitative home and gaming technologies [7]. They aim to support the rehabilitative path directly at home, reducing the need to move towards specialized centers and at the same time, making the exercises more enjoyable.

Based on the above, this paper presents a patented wireless hand motion recognition system called SensHand V1 [8] based on 9-axis complete inertial modules and dedicated microcontrollers which are fixed on fingers and forearm. The technological solution presented is able to track the patients' hand motions in real-time and then to send data through wireless communication. This reduces the clutter and the disadvantages of a glove equipped with sensors through a different structure composed by miniaturized modules to be worn on the distal phalanx. The technological advantages of SensHand V1 can be grouped into different properties, both physical and linked to the type of measurement that the device is able to realize. SensHand V1 is a miniaturized and light structure, easy to wear and to use, independent from the physical build of the person wearing it and from artifacts caused by the movement which make the system very suitable for remote rehabilitation and self monitoring. On the another hand the system is not affected by problems related to the deterioration of the sensors and it does not require frequent calibrations thanks to the type of sensors that provide an intrinsic compensation typical of tri-axial systems. As regard the additional properties, SensHand V1 is characterized by low 
cost components and low power consumption as well as long life thanks to low liability to deterioration.

SensHand V1 has been tested in the study of Parkinson's disease (PD) making a step forward in comparison to previous works aimed at the acquisition of sources of tremor [9] and, generally, at carrying out a quantitative evaluation of the disease [10].

The purpose of this kind of studies is generally to develop different devices to provide quantitative motor symptom severity scores highly correlated to clinical rating scales and to achieve a significant comparison of the motor performances between healthy volunteers and PD patients. These devices are generally wearable (placed on the tip of the index finger in particular) and they consist of only a gyroscope [12], tri-axis accelerometers [14], both of them [11] and/or additional sensors, such as touch sensors [13]. The performance of motor tasks, based on the MDS-UPDRS III motor examination (such as finger tapping, hand-grasping, opening/closing of the hand, pronation-supination of the forearms, evaluation of rest, postural and kinetic tremor), was required to subjects involved in these researches. During the tests, the signals from the sensors were recorded and then amplified, sampled and transmitted to a PC to be processed. Data feature extraction was achieved in the spatio-temporal domain (average speed, amplitude and rhythm ([11],[14]), RMS angular velocity and RMS angular displacement [12], maximum opening velocity and total distance of finger tapping movement [13]) and in the frequency domain (peak power and total power intensity of the movement [12]). Extracted indices showed significant differences between control and patients and also correlations with the clinical scores. Generally healthy people showed more constant repetitive movements, higher frequencies and mean values as well as lower standard deviations related to patients.

\section{INSTRUMENTS}

The SensHand V1 is a wearable sensor system composed, in the current version, of four inertial modules. Each module is able to measure metrics and parameters related to posture, orientation and movement of the human hand through the presence of complete 9-axis inertial sensors (6-axis geomagnetic module LSM303DLHC and 3-axis digital gyroscope L3G4200D, STMicroelectronics, Italy) that guarantee the mapping of motion in the three-dimensional space. In addition, each module includes a microcontroller (ARM®-based 32-bit STM32F10RE MCU, STMicroelectronics, Italy) to acquire, filtering and store data at a frequency of $100 \mathrm{~Hz}$. The coordination of the different modules and the data synchronization is implemented through the Controller Area Network (CAN) standard. One of the SensHand V1 modules plays the role of coordinator of the entire system (Fig.1) collecting the data and transmitting them towards a generic control station through a wireless communication based on the ESD 210 (Parani) Bluetooth serial device. The coordinator is placed on the forearm while the other modules are positioned on the distal phalanx of the thumb, index and middle finger (Fig.2). The device is powered by a small, rechargeable and light Li-Ion battery that ensures

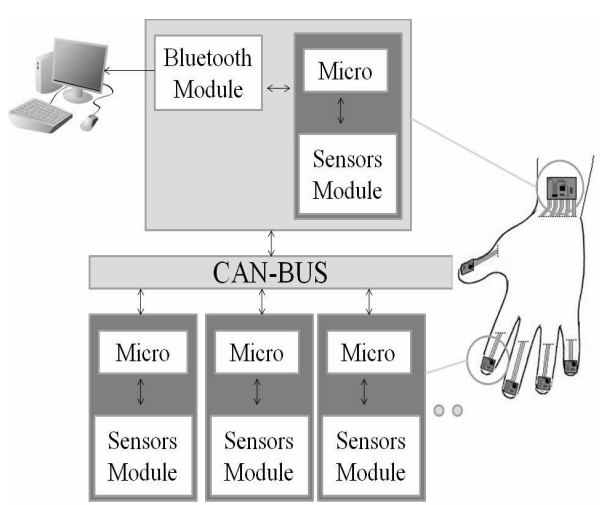

Fig. 1. Block diagram of SensHand V1

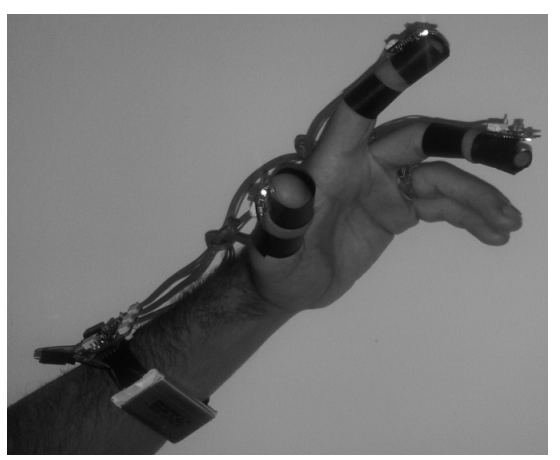

Fig. 2. Image of the prototypal device.

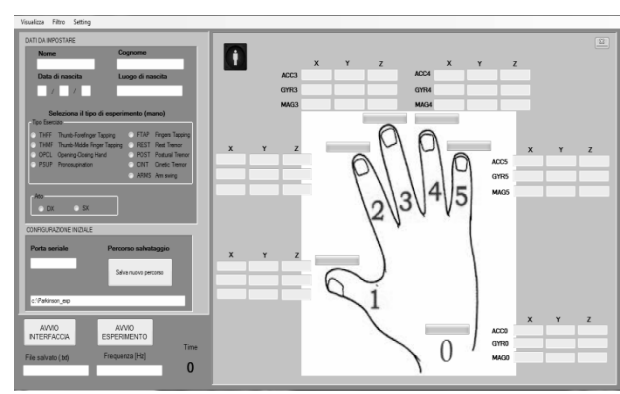

Fig. 3. Interface for sensor data management.

the continuous working of the system for about four hours. A dedicated interface (Fig.3) completes the system to manage the transmission of sensors data, to verify the accuracy of the measures and to store the results for following motion analysis. The interface is programmed to draft individual reports for each acquisition conducted.

\section{METHODOLOGY}

The proper functioning of the whole system was tested through an specific protocol of biomechanical exercises carried out by PD patients and healthy volunteers under the supervision of neurologists. 


\section{A. Recruited Subjects}

Ten patients with Parkinson's disease (PD) (9 men, 1women; mean $67,9 \pm 6,9$ years old) and five healthy controls ( 2 men, 3 women, mean $64,8 \pm 9,1$ years old) were asked to participate in this study. Exclusion criteria were impairments or diseases other than PD (i.e., orthopedic or neurologic) that could affect the performance of experimentation. All subjects lived independently in the community and gave written informed consent prior to the study, whose procedures were approved by the Medical Ethical committee of ASL1 (Azienda Sanitaria Locale, Carrara, Italy).

\section{B. Clinical Assessment}

Patients were preliminarily evaluated by a neurologist using the UPDRS scale as reported in Table I. Particular attention was pointed on movements of hands and tremor (task number: $3.4,3.5,3.6,3.15,3.17,3.18)$.

Table I: Clinical score for the 10 PD patients, with mean and standard deviation of the entire group.

\begin{tabular}{|c|c|c|c|c|c|c|}
\hline ID & 01MA & 02MD & 03NP & 04FA & 05GM & 06OT \\
\hline UPDRS & 61 & 9 & 20 & 27 & 55 & 30 \\
\hline ID & $07 \mathrm{PV}$ & $08 \mathrm{~PB}$ & 09PC & $10 \mathrm{SC}$ & MEAN & SD \\
\hline UPDRS & 21 & 47 & 60 & 79 & 40.9 & 22.7 \\
\hline
\end{tabular}

\section{Experimental protocol}

The experimental protocol for the analysis of movements of the upper limbs was composed by six exercises: pronationsupination of the forearms (PSUP), opening/closing of the hands (OPCL), thumb-forefinger tapping (THFF), thumbmiddle finger tapping (THMF), tremor at rest (REST), postural tremor (POST). They corresponded to the MDS-UPDRS III (tasks: $3.4,3.5,3.6,3.15,3.17,3.18)$. Subjects were asked to sequentially perform the six exercises for five times and for both upper limbs to complete the experimental session. In addition, every subjects made a short preliminary training to try all required movements.

\section{Description of exercises}

During the execution of the exercises, the subjects sat on the chair, holding right angles between trunk and thigh (at the hip) and between thigh and shin (at the knee). The subject's back was leant on the backrest and the not-used arm was held on the armrest in order to have a comfortable posture. Three fingers (distal phalanx of thumb, forefinger and middle finger) and the wrist were sensorised. The six performed exercise are here now described in details:

1) Pronation/supination (PSUP): the subject was directed to assume a sitting posture at rest and asked to put his sensorized arm outstretched in front of himself, with the wrist stable, the hand in prone position and the fingers outstretched approximately $1 \mathrm{~cm}$ apart from each other. After three seconds in rest position, subject executed pronation-supination movements of the forearm as fast and as wide as possible for 10 seconds.

2) Opening/closing hand (OPCL): the subject was directed to assume a sitting posture at rest with sensorized arm flexed at the elbow. The elbow was fixed on the table keeping the palm of the hand in front of the subject. After three seconds in rest position, subject alternatively opened and closeed his/her sensorised hand as fast and as wide as possible for 10 seconds keeping the forearm and the wrist fixed.

3) Thumb-forefinger tapping (THFF): the subject was directed to assume a sitting posture at rest keeping the hand fixed on the desk, so that the plane between thumb and forefinger together joined was parallel to the desk. In the starting position, the thumb and the forefinger were in contact. Subject remained for three seconds in rest position, then he/she tapped his/her forefinger against his thumb as quickly and as widely as possible for 10 seconds.

4) Thumb-middle finger tapping (THMF): the subject was directed to assume a sitting posture at rest keeping the hand fixed on the desk, so that the plane between thumb and middle finger together joined was parallel to the desk. In the starting position, the thumb and the middle finger were in contact. Subject remained for three seconds in rest position, then he/she tapped his/her middle finger against his thumb as quickly and as widely as possible for 10 seconds.

5) Rest Tremor (REST): the subject was directed to assume a sitting posture at rest and was asked to put his/her sensorised hand on the table in prone position, with fingers approximately $1 \mathrm{~cm}$ apart from each other with the elbow on the table. He remained in rest position for the whole duration of the exercise keeping the hand in full relax (he must not to contrast the eventual tremor).

6) Postural Tremor (POST): subject was directed to assume a sitting posture at rest and was asked to put his/her sensorized arm outstretched in front of himself with wrist stable, the hand in prone position and the fingers outstretched approximately $1 \mathrm{~cm}$ apart from each other. He remained in rest position for the whole duration of the exercise.

\section{E. Extracted features}

For each exercise that subjects performed using the SensHand V1, some biomechanical parameters were measured (Table II): frequency, amplitude and smoothness of the movements. So the exercises extracted from the UPDRS scale were evaluated in an objective way by means of biomechanical parameters processing, overtaking the subjectivity of medical judgment.

The inertial data acquired with the SensHand V1 were stored on the PC and offline processed using Matlab. All the measured parameters were obtained from the acceleration and angular rate data. A fourth-order low-pass digital Butterworth filter was applied with $5 \mathrm{~Hz}$ cut-off frequency $\left(f_{c}\right)$ to eliminate high-frequency noise and tremor frequency in the exercises of movement. Only for the tremor exercises, a fourth-order band- 
pass $(1-15 \mathrm{~Hz})$ digital Butterworth filter was applied to eliminate the continuous component and the high-frequency noise in order to appreciate the variations due to the tremor.

In the analysis conducted in the spatio-temporal domain, appropriate algorithms of segmentation were implemented to identify the typical phases for each exercise. Angular rates were integrated using the trapezoidal rule to calculate the amplitude of the movements. For finger tapping exercises, we hypothesized that movement occurred only at the metacarpophalangeal joint.

Table II - Biomechanical features extracted by the different exercises.

\begin{tabular}{|c|c|}
\hline Exercises & Biomechanical Extracted Features \\
\hline$\underline{\text { PSUP }}$ & $\begin{array}{l}\text { - Number of rotations, frequency (rotations/s) } \\
\text { and RMSE of frequency; } \\
\text { - Angular excursion in rotation (deg) and RMSE } \\
\text { of excursion; } \\
\text { - Velocity in supination movement (deg/s) } \\
\text { - Velocity in pronation movement (deg/s) }\end{array}$ \\
\hline OPCL: & $\begin{array}{l}\text { - Number of movements, frequency } \\
\text { (movements/s) and RMSE of frequency; } \\
\text { - Opening hand velocity (deg/s) } \\
\text { - Closing hand velocity (deg/s) } \\
\text { - Angular excursion (deg) and its RMSE; }\end{array}$ \\
\hline THFF & $\begin{array}{l}\text { - Number of taps, frequency (taps/s) and RMSE } \\
\text { of frequency; } \\
\text { - velocity (deg/s) and amplitude (deg) of index } \\
\text { finger movement } \\
\text { - velocity (deg/s) and amplitude (deg) of thumb } \\
\text { movement }\end{array}$ \\
\hline$\underline{\text { THMF }}$ & $\begin{array}{l}\text { - Number of taps, frequency (taps/s) and RMSE } \\
\text { of frequency; } \\
\text { - velocity (deg/s) and amplitude (deg) of middle } \\
\text { finger movement } \\
\text { - velocity (deg/s) and amplitude (deg) of thumb } \\
\text { movement }\end{array}$ \\
\hline$\underline{\text { REST }}$ & $\begin{array}{l}\text { - Dominant Frequency and Ratio between the } \\
\text { Average Power of the signal in the band 3-8 Hz } \\
\text { and the Total Average Power of the signal. } \\
\text { - Ranges of movement for each direction }\end{array}$ \\
\hline$\underline{\text { POST }}$ & $\begin{array}{l}\text { - Dominant Frequency and Ratio between the } \\
\text { Average Power of the signal in the band 8-12 } \\
\text { Hz and the Total Average Power of the signal. } \\
\text { - Ranges of movement for each direction }\end{array}$ \\
\hline
\end{tabular}

\section{EXPERIMENTAL RESULTS}

Generally healthy subjects (in black) showed better performance than patients (in light gray), as illustrated in Figure 4. In particular, in the exercise of Prono-Supination of the forearms the velocity of the movements in controls was higher than in patients. Analogous trend was evident for the frequency of movement in the exercises of Opening-Closing of the hands, Thumb-Forefinger tapping and Thumb-Middle Finger tapping. Healthy subjects in general had higher values in velocity and frequency and lower values in standard deviations and coefficient of variation, showing also a significant statistical difference related to a T-test analysis $(\mathrm{p}<0.05)$. These results confirmed that the typical Parkinson's symptoms, such as bradykinesia and hypokinesia, were responsible for slowing down movements. The analysis of the tremor was carried out in the frequency domain. The signal power in the typical bands of the tremor $(3-8 \mathrm{~Hz}$ and $8-12 \mathrm{~Hz}$ for resting and postural tremor respectively) with respect to the total power of the signal was evaluated. The healthy controls showed a power percentage significantly lower than patients $(p=0.01)$ in the band of frequency $3-8 \mathrm{~Hz}$ for the rest tremor, underlining the difference between the two groups as illustrated in Figure 4. For postural tremor no significant differences $(p>0.05)$ between the two groups have been revealed.

A synthesis of the differences in motor performance between PD patients and healthy controls can be observed in Figure 4 that represents the placement of the subjects in the space of the principal components by means of a PCA.

In Table III the Pearson's coefficients between clinical scores and the extracted motion parameters presented a good correlation $(k>0.7)$ in the majority of cases.

Table III - Correlation between clinical score and significant biomechanical features.

\begin{tabular}{|c|c|c|c|c|c|}
\cline { 2 - 6 } \multicolumn{1}{c|}{$\begin{array}{c}\text { Pearson's } \\
\text { coefficients }\end{array}$} & \multicolumn{5}{|c|}{ Biomechanical parameters } \\
\cline { 2 - 6 } & $\omega \_p s$ & $\omega o \_o p c l$ & f_TF & f_TM & $\begin{array}{c}\text { PWR\% } \\
\text { REST }\end{array}$ \\
\hline $\begin{array}{c}\text { UPDRS } \\
\text { I-IV }\end{array}$ & -0.77 & -0.88 & -0.81 & -0.85 & -0.13 \\
\hline
\end{tabular}

\section{DISCUSSIONS}

This paper proposed an innovative system, SensHand V1, that was used to assess motor performance in PD patients. This system was designed as a modular solution that guaranteed full wearability without any movement artifact. Similar solutions in literature or on the market for hand movement analysis ([16], [17]), such as smart gloves, have limitations in terms of wearability, which leads to low accuracy of measurements and necessity of frequent calibrations. SensHand V1 is an universal solution which reduces the necessity of systems of different sizes through miniaturized modules (about $16 \mathrm{~mm}$ x $18 \mathrm{~mm}$ for the finger board and $43 \mathrm{~mm} \times 32 \mathrm{~mm}$ for the forearm board) adaptable to both hands. The device allows to manipulate objects with different shape, preserving the tactile perception. 

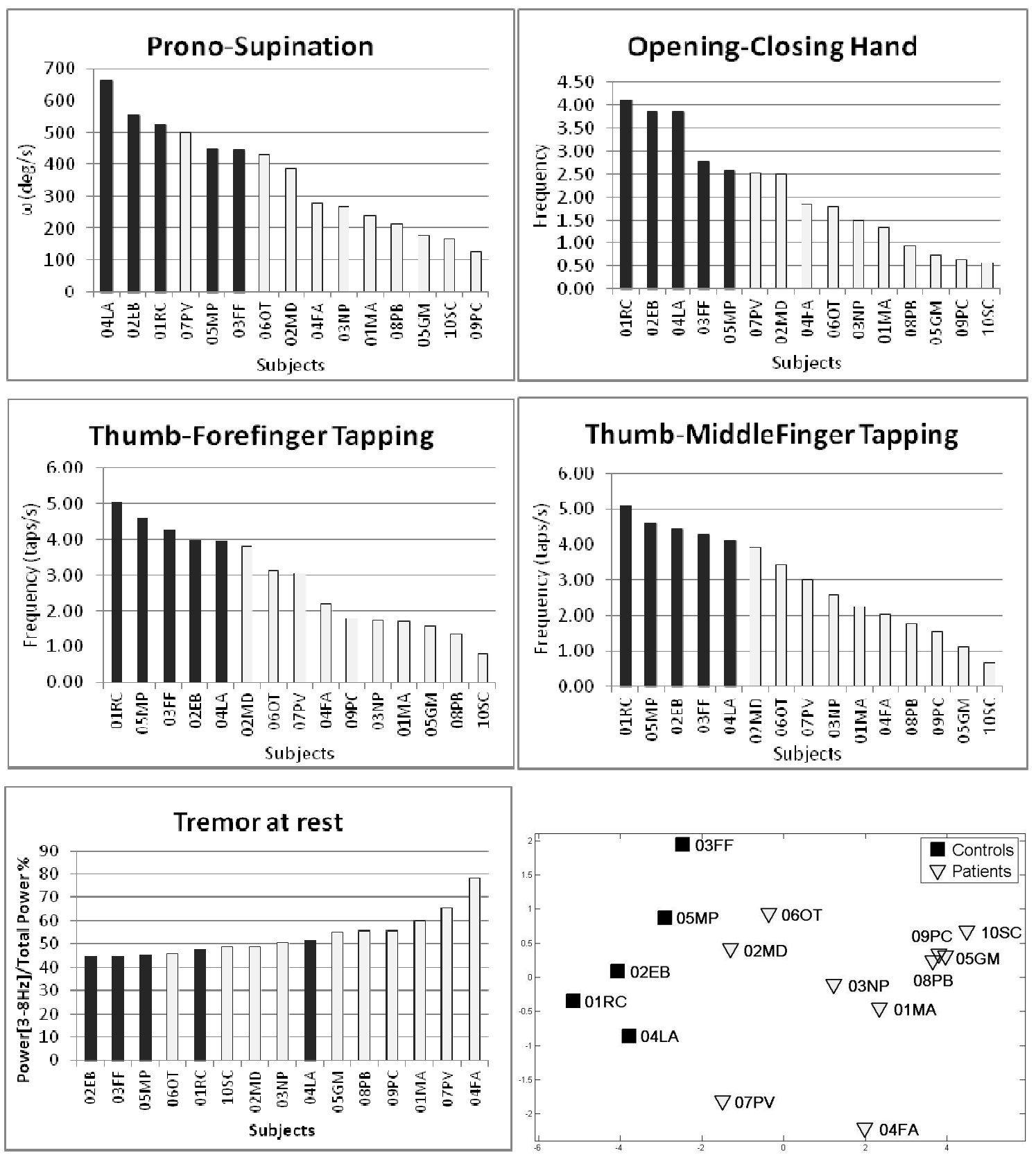

Fig. 4. Performance of subjects during the experimental phase and PCA of PD patients' and healthy controls' motor performances.

\section{CONCLUSIONS AND FUTURE WORKS}

In this paper, a system for hand motion analysis, based on inertial sensors was described. Thanks to the collaboration of ASL1 (Carrara, Italy), SensHand V1 was tested on ten PD patients and five controls to assess the proper functionalities as well as the accuracy and reliability of the measures. A careful analysis of sensor data was carried out to evaluate the performance of the users and to make a direct comparison between healthy subjects and PD patients. The presented technological solution showed a clear potentiality to positively influence the study of Parkinson's disease from the early diagnosis to the disease progression but also from the point of view of industrial interest because this system can find applications in various segments. A new version is already in progress with the idea to design a complete wireless solution, eliminating the cabling between the various modules with the aim to improve the comfort and wearability of the device through the development of a new system characterized by a higher modularity.

\section{ACKNOWLEDGMENT}

This work was supported by the Lundbeck, Denmark. 


\section{REFERENCES}

[1] S.A. Dalley, H. A. Varol, M.Goldfarb, "A Method for the Control of Multigrasp Myoelectric Prosthetic Hands", IEEE TRANSACTIONS ON NEURAL SYSTEMS AND REHABILITATION ENGINEERING, VOL. 20, NO. 1, JANUARY 2012.

[2] N. Tanyawiwat, S. Thiemjarus, "Design of an Assistive Communication Glove using Combined Sensory Channels", 2012 Ninth International Conference on Wearable and Implantable Body Sensor Networks.

[3] G. Saggio, " Bend sensor arrays for hand movement tracking in biomedical systems", Advances in Sensors and Interfaces (IWASI), 2011 4th IEEE International Workshop on.

[4] Liquan Guo, Jiping Wang, Qiang Fang, Xudong Gu, Jianming Fu,"Motion Recognition for Unsupervised Hand Rehabilitation Using Support Vector Machine", Biomedical Circuits and Systems Conference (BioCAS), 2012 IEEE.

[5] Zhiqiang Luo, Chee Kian Lim, I-Ming Chen, Song Huat Yeo,"A virtual reality system for arm and hand rehabilitation", Frontiers of Mechanical Engineering Volume 6, Issue: 1, March, 2011.

[6] E. Szelitzky, A.M. Aluţei, B. Chetran, D. Mândru, "Data Glove and Virtual Environment - a Distance Monitoring and Rehabilitation Solution", Proceedings of the 3rd International Conference on E-Health and Bioengineering - EHB 2011, 24th-26th November, 2011, Iaşi, Romania.

[7] M. Huber, B. Rabin, C. Docan, G. C. Burdea, M. AbdelBaky, M. R. Golomb ,"Feasibility of Modified Remotely Monitored In-Home Gaming Technology for Improving Hand Function in Adolescents With Cerebral Palsy", IEEE TRANSACTIONS ON INFORMATION TECHNOLOGY IN BIOMEDICINE, VOL. 14, NO. 2, MARCH 2010.

[8] Cavallo F., Maremmani C., Esposito D., Rovini E., Aquilano M., Dario P., Carrozza M.C, "Not-glove inertial sensor system for movement analisys of hand: SensHand V1", Italian patent pending, 14/01/2013.

[9] R. Vinjamuri, D.J. Crammond, D. Kondziolka, Heung-No Lee, ZhiHong Mao, "Extraction of Sources of Tremor in Hand Movements of Patients With Movement Disorders", IEEE TRANSACTIONS ON INFORMATION TECHNOLOGY IN BIOMEDICINE, VOL. 13, NO. 1, JANUARY 2009.

[10] K. Niazmand, K. Tonn, A. Kalaras, U.M. Fietzek, J.H. Mehrkens, T.C. Lueth, "Quantitative Evaluation of Parkinson's Disease using sensor based smart Glove", Computer-Based Medical Systems (CBMS), 2011 24th International Symposium on.

[11] T. O. Mera, D. A. Heldman, A. J. Espay, M. Payne, J. P. Giuffrida "Feasibility of home-based automated Parkinson's disease motor assessment", Journal of Neuroscience Methods 203 (2012) 152-156

[12] J.W. Kim, J.H. Lee, Y. Kwon, C.S. Kim, G.M. Eom, S.B. Koh, D.Y. Kwon, K.W. Park; "Quantification of bradykinesia during clinical finger taps using a gyrosensor in patients with Parkinson's disease", Med Biol Eng Comput 2011, 49: 365-371.

[13] M. Yokoe, R. Okuno, T. Hamasaki, Y. Kurachi, K. Akazawa, S. Sakoda, "Opening velocity, a novel parameter, for finger tapping test in patients with Parkinson's disease", Parkinsonism and Related Disorders 15 (2009) 440-444.

[14] J. Stamatakis, J. Cremers, B. Macq and G. Garraux , "Finger tapping feature extraction in Parkinson's disease using low cost accelerometers", 10th IEEE International Conference on Information Technology and Applications in Biomedicine (ITAB), 2010, 3-5 November.

[15] S. Patel, K. Lorincz, R. Huges, N. Huggins, J. Growdon, D. Standaert, M. Akay, J. Dy, M. Welsh, P. Bonato, "Monitoring motor fluctuations in patients with Parkinson's disease using wearable sensors", IEEE transactions on Information Technology in Biomedicine, 2009, Vol.13, N. 6, 864-871.

[16] [Online].Available: http://www.cyberglovesystems.com/.

[17] [Online].Available: http://www.5dt.com/. 